\title{
OVARIAN VEIN THROMBOSIS AND MIRROR SYNDROME IN ASSOCIATION WITH SACROCOCCYGEAL TERATOMA
}

doi: $10.1590 / \mathrm{S1807-59322010000400017}$

\author{
Hasan Kafali MD, ${ }^{\mathrm{I}}$ Yüksel Arıkan Onaran, ${ }^{\mathrm{I}}$ Esra Keskin, ${ }^{\mathrm{I}}$ Umut Sarı, ${ }^{\mathrm{I}}$ Ismail Kırbas ${ }^{\mathrm{II}}$
}

\section{INTRODUCTION}

Ballantyne's syndrome has originally been described for hydrops fetalis, which is associated with rhesus isoimmunization; however, hydrops fetalis can also occur in association with non-immunological causes, including Ebstein's anomaly, Galen's vein aneurysm, fetal arrhythmias, and sacrococcygeal teratoma (SCT). SCT is the most commonly presenting tumor in newborn babies, occurring in approximately 1 in every 40,000 live births. Large or rapidly growing tumors are highly vascular, and lead to high-output cardiac failure, which is characteristic of hydrops fetalis. In non-immune hydrops fetalis maternal preeclampsia (Mirror syndrome) can also sometimes occur. The clinical manifestations of mirror syndrome are quite varied, and the pathophysiology of this syndrome is poorly understood. ${ }^{1-3}$

Ovarian vein thrombosis (OVT) is a rare complication of pregnancy; however, recognition and treatment of this condition are critical because a delay in diagnosis can lead to significant maternal morbidity. The diagnosis of OVT remains a challenge because there is no known profile of risk factors. ${ }^{4}$ In this manuscript, we describe a case of a rapidly growing SCT that is associated with Mirror syndrome and ovarian vein thrombosis.

\section{CASE REPORT}

A 28-year-old G3P1A1 female was referred to our obstetrics and gynecology department after diagnosis of a fetal SCT with solid and cystic components via routine ultrasound at 16 weeks of gestation. The SCT exhibited

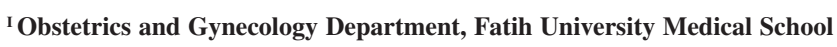
- Çankaya /Ankara, Turkey.

II Radiology Department, Fatih University Medical School - Çankaya / Ankara, Turkey. Email: hasankafali@hotmail.com
}

a cross-sectional area of $5.6 \times 6.4 \mathrm{~cm}^{2}$ and there was also evidence of hydronephrosis and bladder compression. There was no evidence of fetal hydrops fetalis and the initial echocardiogram was normal. This patient's amniotic fluid index was $18 \mathrm{~cm}$. The next fetal ultrasound at 20 weeks demonstrated placentomegaly and polyhydramnios, crosssectional area of the teratoma was found increased from $5.6 \times 6.4 \mathrm{~cm}^{2}$ to $8.2 \times 9.5 \mathrm{~cm}^{2}$. At 28 weeks, the SCT crosssectional area was observed to have increased to $17 \times 12$ $\mathrm{cm}^{2}$. At this point in time, the fetus exhibited generalized skin and scalp edema, abdominal ascites, and pericardial and pleural effusions. The umbilical cord systolic/diastolic ratio was 5.2 , with only very subtle umbilical venous pulsations. There was no flow reversal evident in the ductus venosus waveform. The middle cerebral artery flow was $59.5 \mathrm{~cm} / \mathrm{sec}$, which is consistent with fetal anemia. Based on an elevation in the middle cerebral artery flow, which is suggestive of fetal anemia, in addition to the ultrasound findings of generalized edema, ascites, and pericardial and pleural effusions, we concluded that the fetus was severely hydropic. After counseling, the decision was made to continue observation and intervene in the pregnancy for maternal benefit only. Two days later after her last visit, the mother was admitted to the hospital due to right lumbar pain and increased lower extremity swelling. Her physical and laboratory examinations showed +++ proteinuria, elevated blood pressure (160/90), and grade I hydronephrosis. Intrauterine ex occurred on the second day of the patient's hospitalization. An $860 \mathrm{~g}$ female infant was delivered at 28 and 4/7 weeks by cesarean delivery with Apgar scores of zero at $1^{\text {th }}$ and $5^{\text {th }}$ minute after delivery

The placenta weighed $980 \mathrm{~g}$. The infant was noted as having marked pitting skin edema, significant pleural effusions, abdominal ascites, and an SCT approximately equal in volume to the remainder of her body (Figure 1).

On the second postoperative day, the mother exhibited 


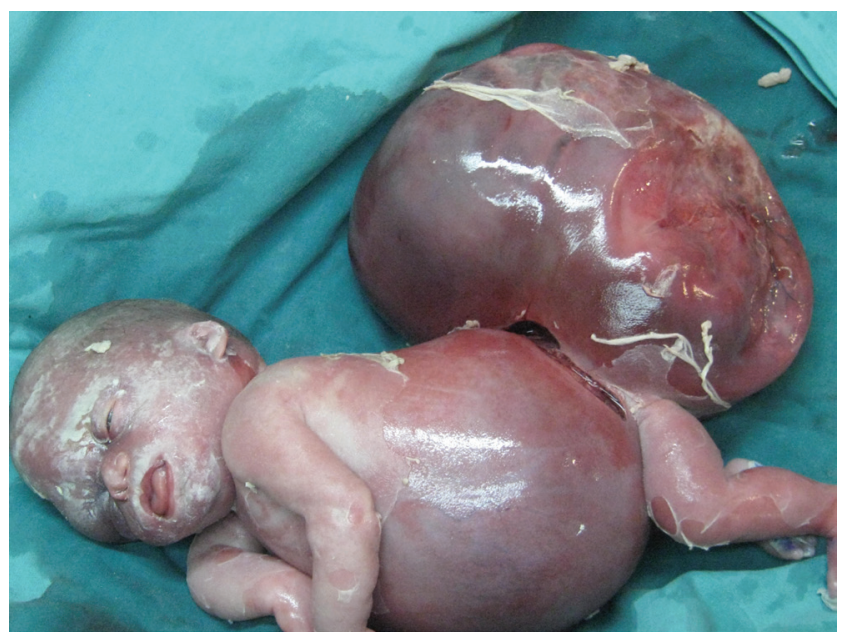

Figure 1 - A view of the huge SCT and the fetus showing hydrops fetalis.

pain in the right iliac fossa and pyrexia $\left(38^{\circ} \mathrm{C}\right)$. An abdominal examination revealed right lower quadrant tenderness that radiated to the ipsilateral flank, rebound tenderness, and right lumbar pain. The patient's white blood cell count was $1100 / \mu 1$, and a blood coagulation profile showed an activated partial thromboblastin time of 37 s (normal value: 25-40), a derived fibrinogen level of $445 \mathrm{mg} / \mathrm{dl}$ (normal value: 200-450), and a D-dimer level of $865 \mu / \mathrm{mm}^{3}$ (normal value: 0-200). An abdominal computed tomographic image exhibited extrinsic compression of the ureter by a hypodense filling defect within the right ovarian vein, which is characteristic of ovarian vein thrombosis and grade III-IV hydronephrosis (Figures 2, 3, and 4). Thus, the patient was treated with a low-molecular weight heparin coagulant (nadroparin $3800 \mathrm{IU}, 0.6 \mathrm{ml}$ every $12 \mathrm{~h}$ ). Three days after the beginning of this therapy, color Doppler ultrasonography revealed a good vascular flow in right ovary, recanalization of the ovarian vein, and a reduction in the amount of fluid in the abdomen. At this point in time, the

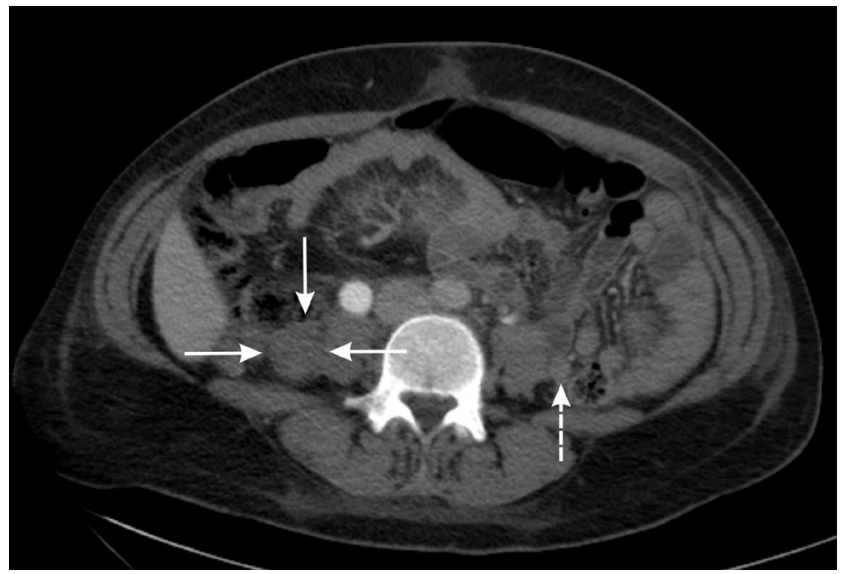

Figure 2 - A computed tomographic image that depicts the patient's normal left ovarian vein (dashed arrow) and right, enlarged and thrombi-filled, ovarian vein (three solid arrows).

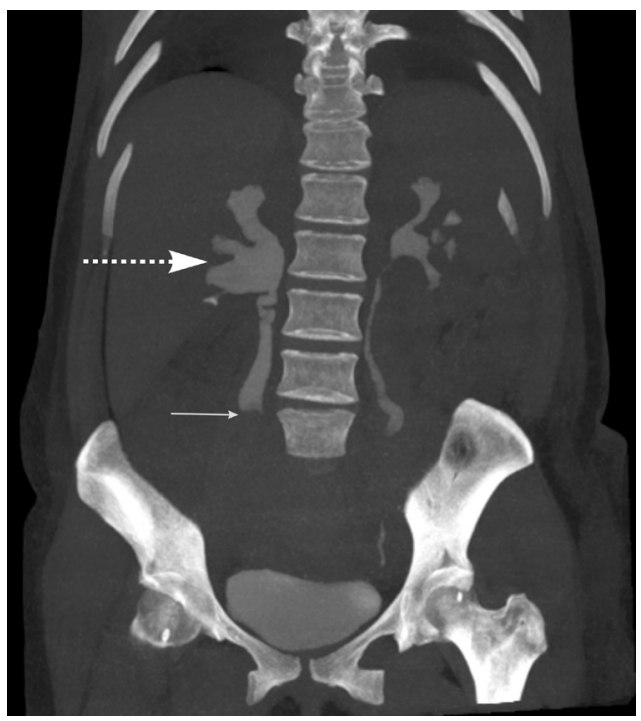

Figure 3 - A computed tomographic image that depicts the obstruction of the right ureter (thin arrow) by the enlarged right ovarian vein (thick dashed arrow).

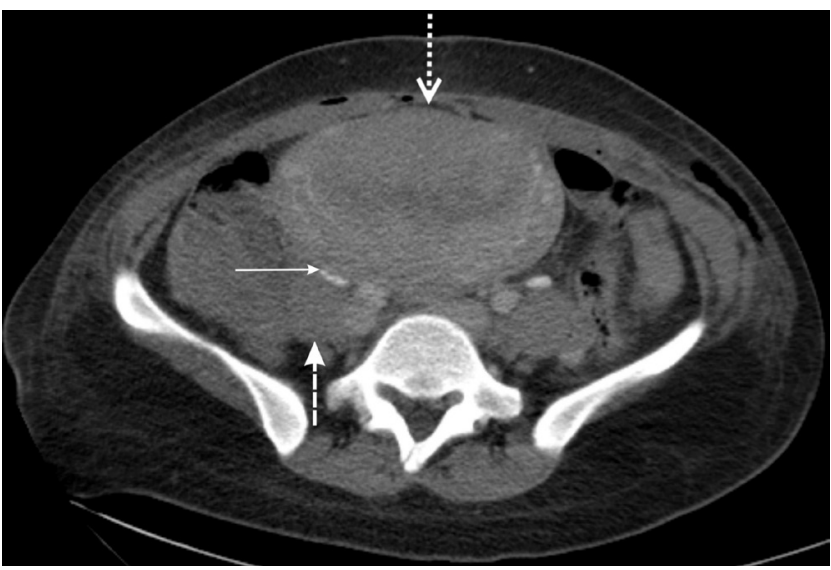

Figure 4 - Grade III-IV hydronephrosis (thick dashed arrow) and ureter obstruction (thin solid arrow).

ureter was still dilated by extrinsic compression; however, a week later, ultrasonography showed normal-size ureter. The patient's coagulation profile was within normal ranges in the following days.

\section{DISCUSSION}

The pathophysiologies of preeclampsia and Mirror syndrome are not clearly understood. Various hypotheses as to the origins of these diseases have been explored, but none have provided exact explanations. Increased oxidative stress in the placental bed in combination with systemic maternal circulation is one of the most popular pathophysiological theories. Several factors that contribute to oxidative stress are plausible as explanations for the pathophysiology of preeclampsia

The first factor involves trophoblast invasion and the 
conversion of maternal spiral arteries into low-resistance vessels that are deficient in preeclampsia. As a result, a risk of residual vascular smooth muscle reactivity with resulting transient hypoxia is hypothesized, which results in an ischemia-reperfusion challenge and increased oxidative stress. ${ }^{5,6}$ Second, debris from necrotic or apoptotic cells may provide proinflammatory stimuli, especially in the presence of a huge placenta mass, such as in the cases of multiple fetuses or molar pregnancy. ${ }^{3}$ We believe that this second mechanism is responsible for the observations made in this case.

In addition, the placental weight was $980 \mathrm{~g}$, which was greater than expected, and there was diffuse hydropic degeneration, which may cause the deportation of more debris than usual to the maternal circulation. An increased inflammatory response to this debris may cause increased oxidative stress and endothelial dysfunction. Endothelial dysfunction results in three processes: impaired homeostasis, an increased risk of thrombosis, and impaired regulation of vascular tone, which clinically presents as hypertension. In fact, as early as 1958, the pathophysiology of preeclampsia was attributed to hyperplacentosis and hypoplacentosis. ${ }^{3}$ In certain types of preeclampsia, as in hydrops fetalis, hydatidiform mole, maternal diabetes, and possibly multiple pregnancies where the placental mass is also often large a state of hyperplacentosis appears to be an essential etiological factor. The main characteristics of hyperplacentosis include a large placental mass with a marked increase in the placental/fetal weight ratio, hydropic chorionic villi, overactivity and immaturity of the trophoblast, and persistence of the Langhans' layer. In other types of preeclampsia, the placenta is small, underactive, and shows evidence of infarction and acceleration of the normal aging process with, presumably, a decrease in functional activity; these anomalies are grouped under the term of hypoplacentosis and generally accompany intrauterine growth retardation. ${ }^{3}$

Ovarian vein thrombosis is a rare, but well-recognized clinical entity that has the potential to cause severe complications. This problem most often occurs in the post-partum period; however, it can also arise in inflammatory disease, after abortion, in association with pre-term or cesarean deliveries, after hysterectomy/ salpingo-oophorectomy, or in the setting of gynecological malignancy. ${ }^{7}$ Other risk factors include systemic lupus erythematosus, antiphospholipid syndrome, the presence of Factor V Leiden, paroxysmal nocturnal hemoglobinuria, hyperhomocysteinemia, $\mathrm{C}$ and $\mathrm{S}$ protein deficiencies, and heparin-induced thrombocytopenia. ${ }^{8-10}$

OVT during pregnancy is a rare pathology that is characterized by inflammation or thrombosis of one or both ovarian veins. The pathogenesis of OVT during pregnancy is still not clearly understood. A state of hypercoagulability during pregnancy, an increased diameter of the ovarian vein, a decrease of blood flow velocity in the ovarian veins following delivery, and hereditary prothrombotic predisposition factors have been considered to be possible causes of OVT. ${ }^{4}$ As was true in the current case, $90 \%$ of OVT cases occur in the right ovarian vein. This predominant right localization is partly due to the dextrotorsion of the puerperal enlarged uterus, which causes compression of the ovarian vein or of the incompetent valves of the right ovarian vein that induce blood flow stasis. Furthermore, the right ovarian vein is also longer than the left, and its incompetent valves are a nidus for thrombosis. ${ }^{4}$

The clinical presentation of OVT is similar to that of acute appendicitis, wherein the latter is the suspected diagnosis in most cases. Fever is seen in $80 \%$, right iliac fossa pain in $55 \%$, and lower left quadrant pain in $3.6 \%$ of patients. ${ }^{11}$ In addition to appendicitis, OVT is often difficult to distinguish from endometritis, hydronephrosis, right-sided ureteral obstruction, hydronephritis, and other ovarian diseases, such as torsion. A high index of suspicion and the ability to rule out other processes that may produce similar symptoms is important, as untreated OVT can have catastrophic consequences and may even prove to be fatal. ${ }^{12}$

Our patient suffered from lower quadrant and flank pain without fever, as well as grade I hydronephrosis of the right kidney since $28^{\text {th }}$ week of pregnancy. During the postpartum period, her complaint and grade of hydronephrosis was aggravated, and she exhibited a fever despite receiving a broad-spectrum antibiotic. Based on these data, we think that the process of ovarian vein thrombosis began during pregnancy and was aggravated after delivery. During the postpartum period, the question of a hereditary prothrombotic predisposition was explored in our case, but none was found; however, we strongly believe that the ovarian vein compression by the huge SCT, in addition to an enlarged uterus, increased the prothrombotic predisposition because preeclampsia may contribute to the existence of OVT.

After an extensive literature search, two cases that associated Mirror syndrome to $\mathrm{SCT}^{1,13}$ and no cases of associations between Mirror syndrome, ovarian vein thrombosis, and SCT were found. Knowledge of these syndromes and clinical suspicion in pregnant patients, whose fetuses show hydrops fetalis, proteinuria, and hypertension, and in puerperal patients suffering from lower quadrant pain and fever (which alone does not respond to adequate broadspectrum antibiotics), should serve as guides for appropriate diagnosis. 


\section{REFERENCES}

1. Finamore PS, Kontopoulos E, Price M, Giannina G, Smulian JC. Mirror syndrome associated with sacrococcygeal teratoma: a case report. J Reprod Med. 2007;52:225-7.

2. Midgley DY, Harding K. The Mirror syndrome. Eur J Obstet Gynecol Reprod Biol. 2000;88:201-2.

3. Carbillon L, Oury JF, Guerin JM, Azancot A, Blot P. Clinical biological features of Ballantyne syndrome and the role of placental hydrops. Obstet Gynecol Surv. 1997;52:310-4.

4. Kominiarek MA, Hibbard JU. Postpartum ovarian vein thrombosis: an update. Obstet Gynecol Surv. 2006;61:337-42.

5. Cheng MH, Wang PH. Placentation abnormalities in the pathophysiology of preeclampsia. Expert Rev Mol Diagn. 2009;9:37-49.

6. Gilbert JS, Ryan MJ, LaMarca BB, Sedeek M, Murphy SR, Granger JP. Pathophysiology of hypertension during preeclampsia: linking placental ischemia with endothelial dysfunction. Am J Physiol Heart Circ Physiol. 2008;294:541-50.

7. Yildirim E, Kanbay M, Ozbek O, Coskun M, Boyacioglu S. Isolated idiopathic ovarian vein thrombosis: A rare case. Int Urogynecol J. 2005;16:308-10.
8. Al-toma A, Heggelman BGF, Kramer MHH. Postpartum ovarian vein thrombosis: Report of a case and review of literature. Neth J Med. 2003;61:334-6.

9. Suleyman T, Gultekin H, Abdulkadir G, Tevfik P, Abdulkerim UM, Ali A, et al. Acute right lower quadrant abdominal pain as the presenting symptom of ovarian vein thrombosis in pregnancy. J Obstet Gynaecol Res. 2008 ;34(4 Pt 2):680-2.

10. André M, Delèvaux I, Amoura Z, Corbi P, Courthaliac C, Aumaître O, et al. Ovarian vein thrombosis in the antiphospholipid syndrome. Arthritis Rheum. 2004;50:183-6.

11. Prieto-Nieto MI, Perez-Robledo JP, Rodriguez-Montes JA, GarciaSancho-Martin L. Acute appendicitis-like symptoms as initial presentation of ovarian vein thrombosis. Ann Vasc Surg. 2004;18:481-3.

12. Takach TJ, Cervera RD, Gregoric ID. Ovarian vein and caval thrombosis. Tex Heart Inst J. 2005;32:579-82.

13. Ibele A, Flake A, Shaaban A. Survival of a profoundly hydropic fetus with a sacrococcygeal teratoma delivered at 27 weeks of gestation for maternal mirror syndrome. J Pediatr Surg. 2008 ;43:e17-20. 